\title{
Mutational and Combinatorial Control of Self-Assembling and Disassembling of Human Proteasome $\alpha$ Subunits
}

Taichiro Sekiguchi ${ }^{1,2,3,4,+}{ }^{+}$, Tadashi Satoh ${ }^{4,+}$, Eiji Kurimoto ${ }^{5}$, Chihong Song $\left.{ }^{6}{ }^{(}\right)$, Toshiya Kozai ${ }^{7}$, Hiroki Watanabe ${ }^{3}{ }^{(0)}$, Kentaro Ishii ${ }^{3,4}$, Hirokazu Yagi ${ }^{4}{ }^{(0}$, Saeko Yanaka ${ }^{1,2,3,4} \mathbb{C}^{(}$, Susumu Uchiyama ${ }^{3,8}$, Takayuki Uchihashi ${ }^{3,7}$, Kazuyoshi Murata ${ }^{6,9}$ (]) and Koichi Kato $1,2,3,4, *$ (D)

1 School of Physical Science, SOKENDAI (The Graduate University for Advanced Studies), Okazaki, Aichi 444-8787, Japan; sekiguchi@ims.ac.jp (T.S.); saeko-yanaka@ims.ac.jp (S.Y.)

2 Institute for Molecular Science, National Institutes of Natural Sciences, 5-1 Higashiyama, Myodaiji, Okazaki, Aichi 444-8787, Japan

3 Exploratory Research Center on Life and Living Systems (ExCELLS), National Institutes of Natural Sciences, 5-1 Higashiyama, Myodaiji, Okazaki, Aichi 444-8787, Japan; hwatanabe@d.phys.nagoya-u.ac.jp (H.W.); ishii@ims.ac.jp (K.I.); suchi@bio.eng.osaka-u.ac.jp (S.U.); uchihast@d.phys.nagoya-u.ac.jp (T.U.)

4 Graduate School of Pharmaceutical Sciences, Nagoya City University, 3-1 Tanabe-dori, Mizuho-ku, Nagoya 467-8603, Japan; tadashisatoh@phar.nagoya-cu.ac.jp (T.S.); hyagi@phar.nagoya-cu.ac.jp (H.Y.)

5 Faculty of Pharmacy, Meijo University, Tempaku-ku, Nagoya 468-8503, Japan; kurimoto@meijo-u.ac.jp

6 National Institute for Physiological Sciences, National Institutes of Natural Sciences, 5-1 Higashiyama, Myodaiji, Okazaki, Aichi 444-8787, Japan; chsong@nips.ac.jp (C.S.); kazum@nips.ac.jp (K.M.)

7 Department of Physics, Nagoya University, Furo-cho, Chikusa-ku, Nagoya 464-8602, Japan; toshiya.kozai@unibas.ch

8 Department of Biotechnology, Graduate School of Engineering, Osaka University, 2-1 Yamadaoka, Suita, Osaka 565-0871, Japan

9 School of Life Science, SOKENDAI (The Graduate University for Advanced Studies), Okazaki, Aichi 444-8787, Japan

* Correspondence: kkatonmr@ims.ac.jp; Tel.: +81-564-59-5225; Fax: +81-564-59-5224

+ These authors contributed equally to this work.

Received: 27 March 2019; Accepted: 7 May 2019; Published: 9 May 2019

check for updates

\begin{abstract}
Eukaryotic proteasomes harbor heteroheptameric $\alpha$-rings, each composed of seven different but homologous subunits $\alpha 1-\alpha 7$, which are correctly assembled via interactions with assembly chaperones. The human proteasome $\alpha 7$ subunit is reportedly spontaneously assembled into a homotetradecameric double ring, which can be disassembled into single rings via interaction with monomeric $\alpha 6$. We comprehensively characterized the oligomeric state of human proteasome $\alpha$ subunits and demonstrated that only the $\alpha 7$ subunit exhibits this unique, self-assembling property and that not only $\alpha 6$ but also $\alpha 4$ can disrupt the $\alpha 7$ double ring. We also demonstrated that mutationally monomerized $\alpha 7$ subunits can interact with the intrinsically monomeric $\alpha 4$ and $\alpha 6$ subunits, thereby forming heterotetradecameric complexes with a double-ring structure. The results of this study provide additional insights into the mechanisms underlying the assembly and disassembly of proteasomal subunits, thereby offering clues for the design and creation of circularly assembled hetero-oligomers based on homo-oligomeric structural frameworks.
\end{abstract}

Keywords: proteasome; self-assembly; homo-oligomer; hetero-oligomer; size exclusion chromatography; native mass spectrometry; crystal structure; atomic force microscopy; electron microscopy 


\section{Introduction}

Proteins in living systems are often assembled into filamentous and circular oligomers, which exert appropriate biological functions and are deposited as malfunctional aggregates, such as pathological amyloids. Circular assemblages composed of identical protomers give rise to functional barrels or cages, as exemplified by chaperonins and AAA ATPases [1-3]. The design and creation of circular oligomers have been important and challenging issues in protein engineering [4,5]. During evolutionary processes, the building blocks of these homo-oligomers acquire diversity in sequence and structure, maintaining their assembling properties. The proteasome system is one of the best examples demonstrating this concept [6-8].

The proteasome is a huge protein complex harboring a proteolytic chamber termed the $20 \mathrm{~S}$ core particle. This $20 \mathrm{~S}$ core particle comprises the $\alpha$ - and $\beta$-rings, two types of heptameric rings arranged as a cylindrical, four-layered $\alpha \beta \beta \alpha$ structure [6-13]. In the archaea, the heptameric $\alpha$-ring is composed of seven identical $\alpha$ subunits and the heptameric $\beta$-ring is composed of one or two kinds of $\beta$ subunits. A total of 28 subunits are spontaneously assembled into the $20 \mathrm{~S}$ core particle [14]. In contrast, in eukaryotes, both the $\alpha$ - and the $\beta$-rings are heteroheptamers composed of seven different but homologous subunits, i.e., $\alpha 1-\alpha 7$ and $\beta 1-\beta 7$. Assembly of these subunits is not an autonomous process but is assisted by several chaperones operating as molecular matchmakers and checkpoints $[8,11,13,15-21]$.

Among all the human proteasome $\alpha$ subunits, $\alpha 7$ exhibits a unique feature of in vitro self-assembly into a homotetradecameric double-ring structure [22-25], thereby raising the question whether the $\alpha 7$ homotetradecamer is an off-pathway dead-end product of the proteasome formation process. Another possibility is that certain mechanisms exist for disassembling the homo-oligomer of the $\alpha 7$ subunit, thereby resulting in its monomeric form, which is a component of the heteroheptameric $\alpha$-ring.

We previously determined a crystal structure of the human $\alpha 7$ homotetradecamer and demonstrated that $\alpha 6$, which exists as a monomer, interacts with this assemblage, thereby disrupting its double-ring structure $[25,26]$. These findings suggest that different $\alpha$ subunits have different assembly properties. We comprehensively characterized the oligomeric states of the $\alpha$ subunits of the human proteasome and examined their possible interplay for obtaining a better understanding of the design principles underlying the self-assembly and disassembly of the proteasomal subunits, and more generally, those behind formation of circularly assembled hetero-oligomers composed of structurally homologous subunits.

\section{Results and Discussion}

\subsection{Oligomeric States of Human Proteasomal $\alpha$ Subunits}

To characterize the oligomeric states of the $\alpha$ subunits in solution, we performed size-exclusion chromatography (SEC) and native mass spectrometry (MS). SEC data revealed that the major fractions of $\alpha 1, \alpha 2, \alpha 3, \alpha 4, \alpha 5$, and $\alpha 6$ corresponded to monomeric-to-dimeric forms and that $\alpha 7$ formed a significantly larger oligomer, as shown in Figure 1a. These findings were confirmed by native MS, which indicated that $\alpha 7$ existed as a homotetradecamer and that the remaining subunits exhibited one major and one minor ion series, corresponding to the molecular masses of the monomer and dimer, respectively, as shown in Figure 1b. Based on these data and our previously reported data regarding sedimentation velocity analytical ultracentrifugation [25], we concluded that among the seven types of $\alpha$ subunits, only $\alpha 7$ is self-assembled into the homotetradecameric double-ring structure and that the remaining $\alpha$ subunits are under equilibrium between the monomeric and dimeric forms. 
(a)

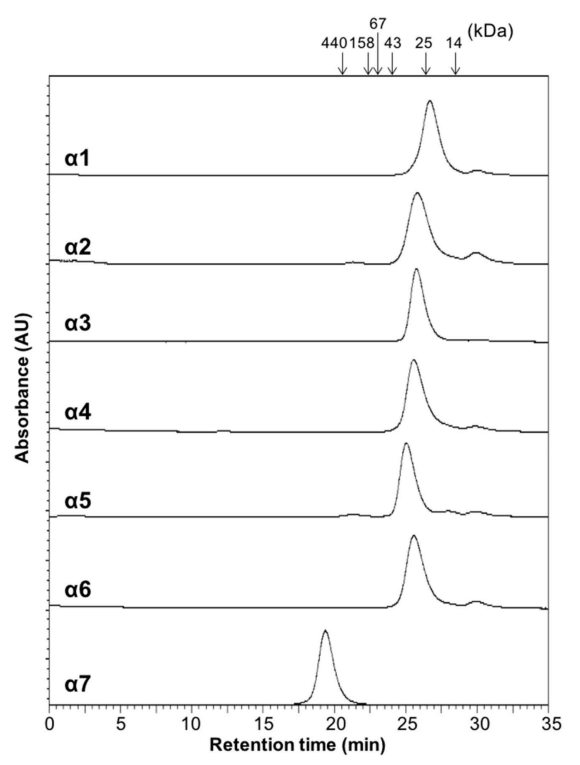

(b)
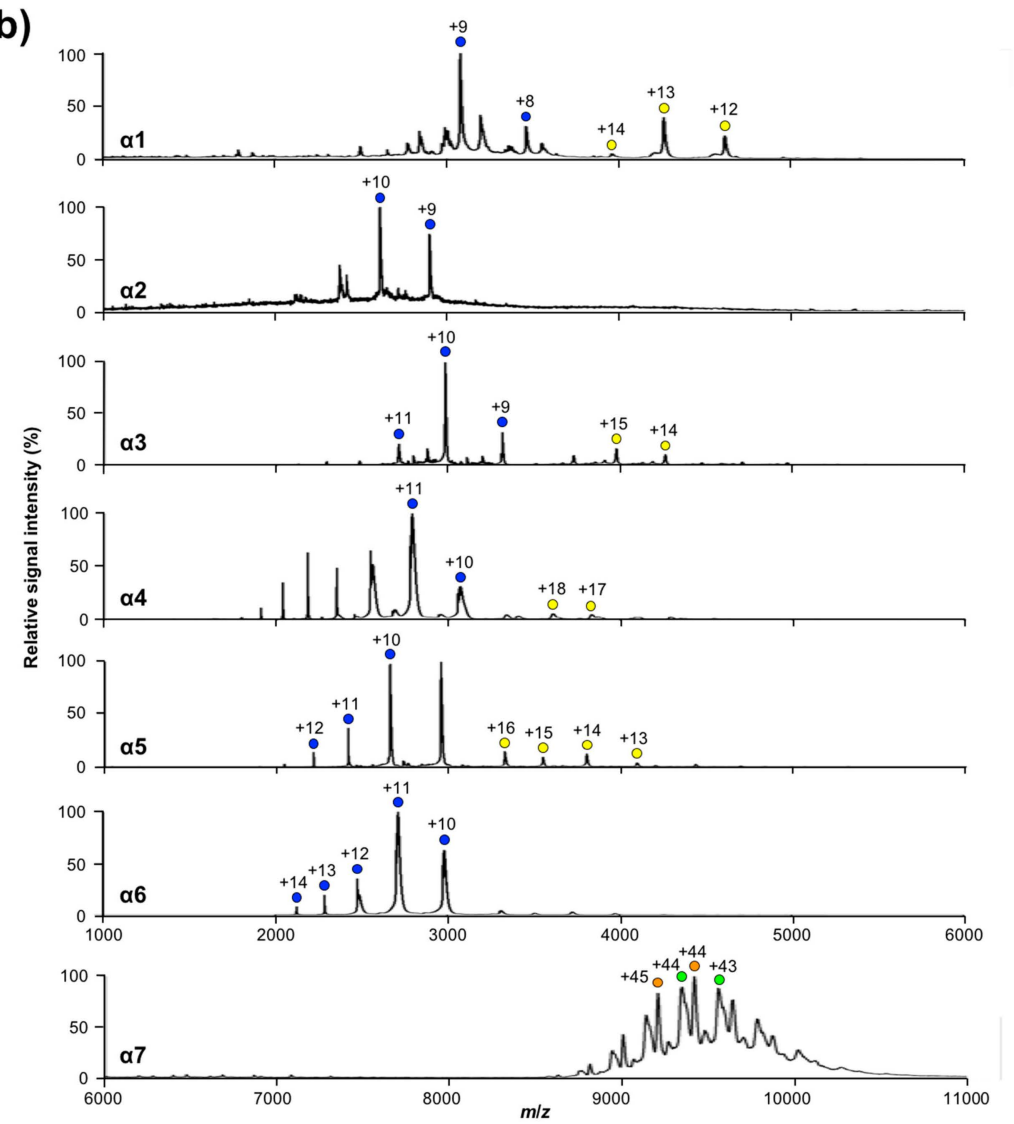

Figure 1. Characterization of the oligomeric states of proteasome $\alpha$ subunits. (a) Size-exclusion chromatogram of $\alpha 1-\alpha 7$ subunits. Arrows indicate eluted positions of the size markers. (b) Mass spectra of $\alpha 1-\alpha 7$ subunits under nondenaturing conditions. Blue and yellow circles indicate the ion series of $\alpha 1-\alpha 6$ monomers and dimers, respectively. Green and orange circles indicate the ion series of the $\alpha 7$ homotetradecamer. The estimated molecular masses of the $\alpha 1-\alpha 7$ subunits are as follows: $27,688.97 \pm 9.91$ and 55,398.59 \pm 3.26 ( $\alpha 1$ dimer and monomer, respectively); 26,034.50 $\pm 4.01(\alpha 2)$; $59,650.89 \pm 6.81$ and $29,818.58 \pm 0.72(\alpha 3) ; 61,304.84 \pm 9.52$ and 30,669.77 $\pm 0.02(\alpha 4) ; 53,162.75 \pm 1.93$ and $26,559.17 \pm 1.39(\alpha 5) ; 29,696.61 \pm 9.90(\alpha 6)$; and $414,406.97 \pm 51.59$ and $411,316.63 \pm 48.20(\alpha 7)$. 


\subsection{Mutational Disassembling of the $\alpha 7$ Homotetradecamer}

To gain insight into the mechanisms of the assembly of $\alpha 7$, we inspected the crystal structure of its homotetradecamer [25]; our inspection highlighted five regions (regions 1-5) mediating intersubunit interactions, as shown in Figure 2. Regions 1, 2, and 3 are involved in intra-ring (cis) interactions, which are shared with the homoheptameric $\alpha$-ring of archaeal $20 S$ core particle $[9,27]$, whereas regions 4 and 5 are involved in inter-ring (trans) interactions. Comparison of amino acid sequences across the human $\alpha$ subunits in terms of these regions revealed that region 1 involves highly conserved contacting pairs of residues (P16-Y25, F14-A29, and F14-P130), whereas most other pairs are unique for $\alpha$, which explains its specific self-assembling property, as shown in Figure S1 and Table S1. Intriguingly, mutational monomerization of an archaeal $\alpha$ subunit (Thermoplasma acidophilum) was achieved by truncating region 1 (residues 2-34), which was accompanied by alanine substitutions of arginine residues (Arg57, Arg86, and Arg130) in regions 2 and 3 [14]. Therefore, we tested whether the conserved residues in region 1 contribute to the formation of the human $\alpha 7$ homo-oligomer using an $\alpha 7$ mutant $\left(\alpha 7^{*}\right)$ in which the N-terminal segment in region 1 (residues 1-22, MSYDRAITVFSPDGHLFQVEYA) was replaced with a hexahistidine-containing segment (MGSSHHHHHHSSGLVPRGSHMGS). SEC and native MS indicated that $\alpha 7^{*}$ was monomeric in solution, as shown in Figure 3, thereby demonstrating that deletion of the $\mathrm{N}$-terminal segment disrupts the homotetradecameric structure. Close inspection of region 1 in the crystal structure of the $\alpha 7$ homotetradecamer highlighted the hydrophobic ball-and-socket joint composed of Phe14 in one subunit fitting into a socket formed by Ala29 and Pro130 in the neighboring subunit, as shown in Figure 2c. A single-mutation F14A disassembled the $\alpha 7$ tetradecamer into the monomeric form, as shown in Figure 3, indicating that the N-terminal segment, particularly Phe14, is critically involved in intra-ring cis interaction as a prerequisite for inter-ring trans interaction. Because Phe14 as well as Ala29 and Pro130 in region 1 are all conserved across $\alpha 1-\alpha 7$, the remaining regions, i.e., regions $2-5$, are likely to reinforce the $\alpha 7$-specific homophilic interaction, thereby stabilizing the tetradecameric complex.

The $\alpha 7$ tetradecamer involves inter-ring interactions mediated through regions 4 and 5 . In contrast, despite the fact that like $\alpha 7, T$. acidophilum formed a double-ring structure in solution, the $\alpha$ subunit of the Archaeoglobus fulgidus proteasome exhibits a single-ring structure in the crystal [14,27]. We previously reported that the $\alpha 7$ single-ring structure can be stabilized on a mica surface and that the $\alpha 7$ tetradecameric double-ring structure is disassembled upon addition of the $\alpha 6$ subunit, thereby forming a 1:7 hetero-octameric $\alpha 6 / \alpha 7$ complex $[25,26]$. These observations suggest that the inter-ring trans interaction is dispensable for stabilizing the heptameric ring structure. We examined this possibility via a mutational approach.

For this purpose, we attempted to introduce electrostatic repulsion at the double-ring interface by focusing on the three autologously contacting pairs Ser96-Ser96, Phe102-Phe102, and Tyr104-Tyr104. Ser96 was substituted with aspartate, whereas Phe102 and Tyr104 were both substituted with arginine, as shown in Figure 4a. In addition to these positions, because the Ser100 positions are spatially proximal to each other across the inter-ring interface, Ser100 was mutated into aspartate. In SEC, the great majority of this quadruple $\alpha 7$ mutant ( $\alpha 7^{\mathrm{SR}}$ ) eluted significantly later (at $21.1 \mathrm{~min}$ ) than the wild-type $\alpha 7$ double ring (at $19.4 \mathrm{~min}$ ), as shown in Figure $4 \mathrm{~b}$. Under nondenaturing conditions, the mass spectrum of this mutant exhibited a major ion series with molecular masses of 207,581 \pm 78 and $209,044 \pm 50 \mathrm{Da}$, corresponding to the heptameric $\alpha 7$ subunits (with a theoretical mass of 199,331 Da), as shown in Figure 4c. In addition, using atomic force microscopy (AFM), we confirmed the heptameric ring of the $\alpha 7$ mutant with a height of $\sim 4 \mathrm{~nm}$, which was half of that of the wild-type $\alpha 7$ double-ring $(\sim 9 \mathrm{~nm})$ [26], as shown in Figure S2. All these data indicate that the double-ring tetradecamer of $\alpha 7$ is disassembled into a single heptameric ring by mutations bringing about electrostatic repulsion at the inter-ring interface, thereby demonstrating that the inter-ring interaction is dispensable for the formation of the homoheptameric ring of $\alpha 7$. 
(a)
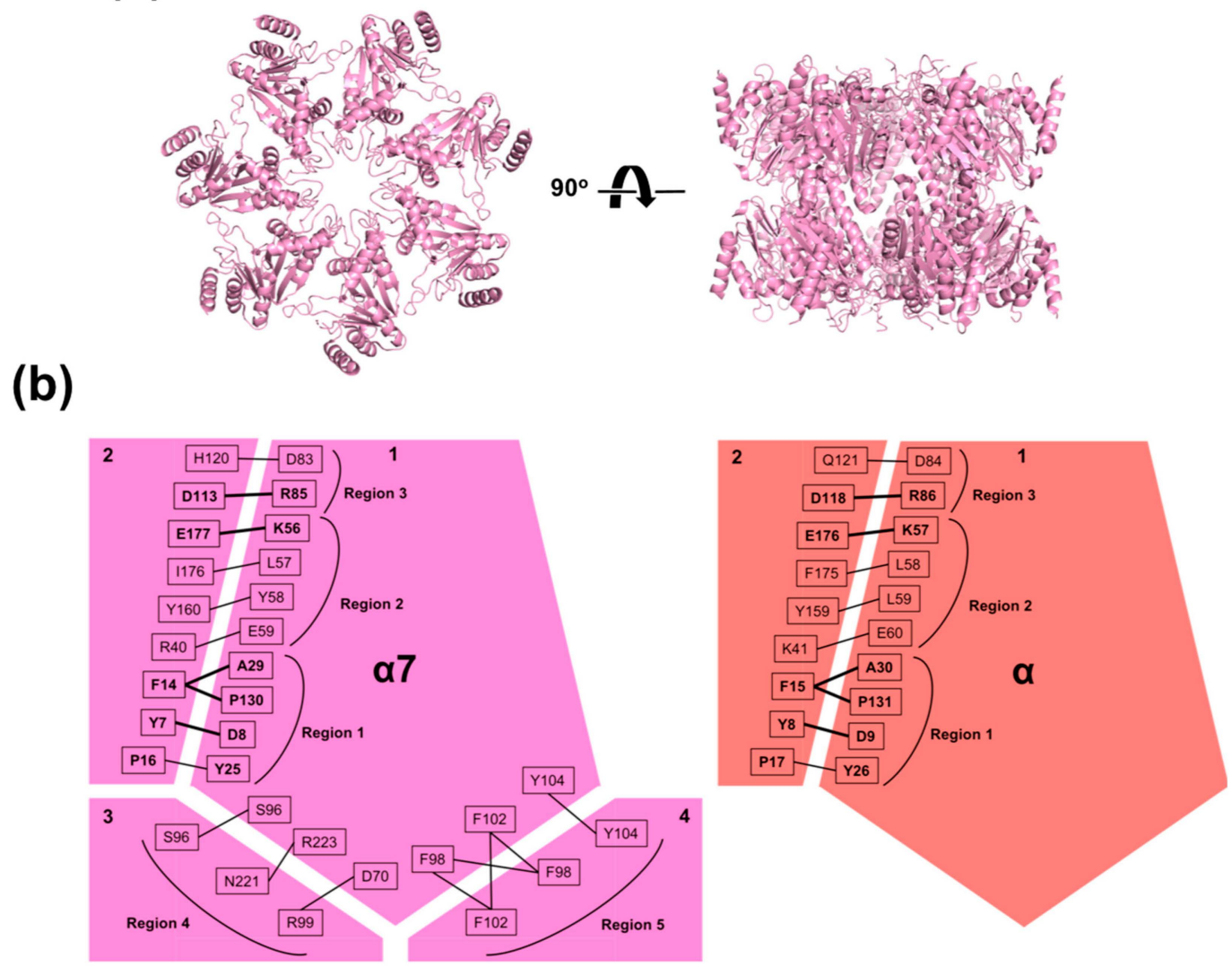

(c)

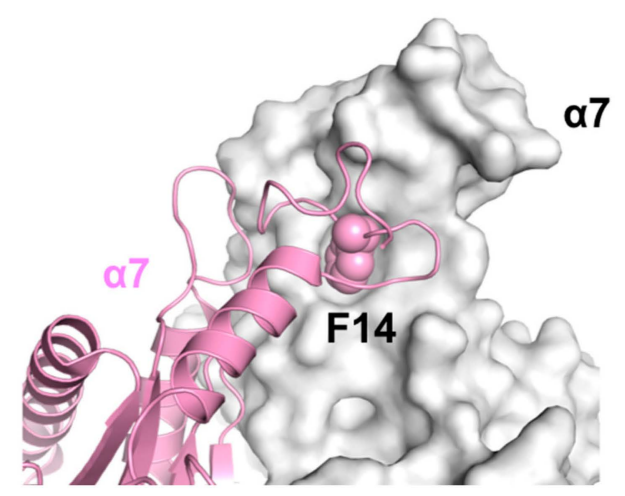

Figure 2. Schematic representation of the binding interfaces of the $\alpha 7$ homotetradecamer and the archaeal $\alpha$ homoheptamer. (a) Crystal structure of the $\alpha 7$ homotetradecamer (Protein Data Bank (PDB) code: $5 \mathrm{DSV}$ ). The left and right structures are related by a rotation of $90^{\circ}$ around the horizontal axis. (b) Schematic representations of intersubunit cis and trans interactions of the human $\alpha 7$ homotetradecamer (adjacent four molecules) together with cis interactions of the Archaeoglobus fulgidus proteasome $\alpha$ homoheptamer (adjacent two molecules, PDB code: 1J2P). Interaction pairs conserved between human $\alpha 7$ and $A$. fulgidus proteasome $\alpha$ subunits are highlighted by bold lines. (c) Surface and ribbon models of intersubunit cis interaction highlighting F14 residue in $\alpha 7$ homotetradecamer. 
(a)

(b)
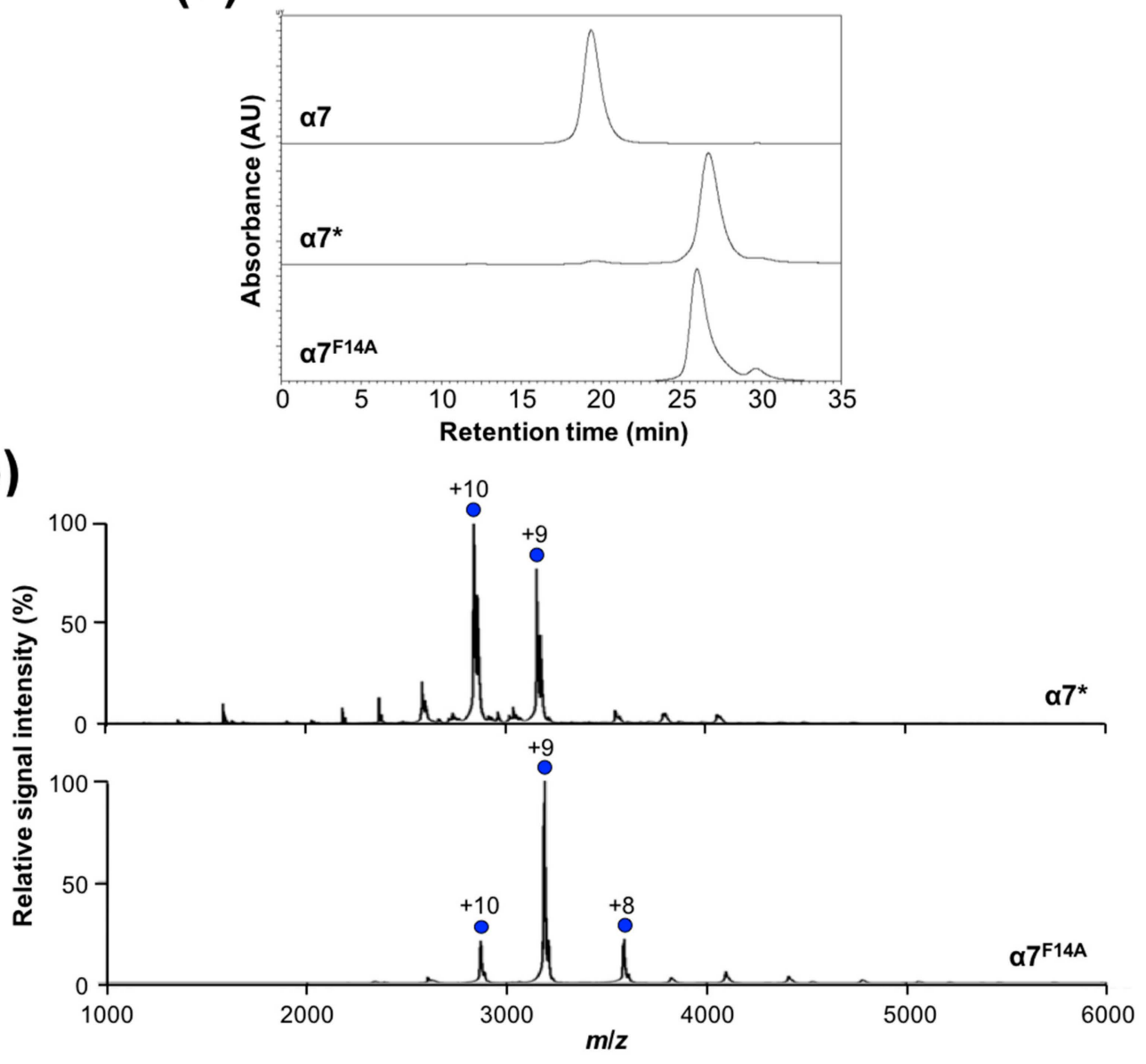

Figure 3. Characterization of the oligomeric states of $\alpha 7$ monomeric mutants. (a) Size-exclusion chromatogram of $\alpha 7^{*}$ and $\alpha 7^{\mathrm{F} 14 \mathrm{~A}}$ together with wild-type $\alpha 7$. (b) Mass spectra of $\alpha 7^{*}$ and $\alpha 7^{\mathrm{F} 14 \mathrm{~A}}$ under nondenaturing conditions. Blue circles indicate the ion series of the $\alpha 7^{*}$ or $\alpha 7^{\mathrm{F} 14 \mathrm{~A}}$ monomer. The mass spectra of $\alpha 7^{*}$ and $\alpha 7^{\mathrm{F} 14 \mathrm{~A}}$ mutants under nondenaturing conditions exhibited the major ion series with molecular masses of the monomer 28,456 \pm 0 (with a theoretical mass: 28,594 Da) and 28,691 \pm 0.05 (28,638 Da), respectively.

\subsection{Disassembly of the $\alpha 7$ Double-Ring via Subunit Interactions}

In addition to being disassembled by mutations, the $\alpha 7$ double-ring structure can be disassembled into its single-ring structure via interaction with the monomeric $\alpha 6$ subunit, thereby forming a 1:7 hetero-octameric $\alpha 6 / \alpha 7$ complex $[25,26]$. We examined whether the other $\alpha$ subunits have such a disassembling capability by performing a comprehensive analysis of the oligomeric states of $\alpha 7$ in the presence of the $\alpha 1-\alpha 6$ subunits along with the monomerized $\alpha 7$ mutant $\alpha 7^{*}$. The SEC data indicated that although neither $\alpha 1, \alpha 2, \alpha 3, \alpha 5$, nor $\alpha 7^{*}$ affected the tetradecameric structure of $\alpha 7$, approximately $20 \%$ and $50 \%$ of the $\alpha 7$ tetradecamer was disrupted into smaller complexes by $\alpha 4$ or $\alpha 6$, respectively, judging from the peak intensity reduction, as shown in Figure $5 \mathrm{a}$. The almost identical elution times (19.7 $\mathrm{min}$ ) between the resultant complexes generated in the $\alpha 4 / \alpha 7$ and $\alpha 6 / \alpha 7$ mixtures implied that like $\alpha 6 / \alpha 7, \alpha 4$ and $\alpha 7$ also form a 1:7 hetero-octameric complex $[25,26]$. To confirm this, we performed native MS of the $\alpha 4 / \alpha 7$ mixture; results indicated that like the $\alpha 6 / \alpha 7$ complex [25], the $\alpha 4 / \alpha 7$ mixture exhibits a major ion series corresponding to a 1:7 hetero-octameric $\alpha 4 / \alpha 7$ complex with a molecular mass of $228,789 \pm 9 \mathrm{Da}$ (theoretical molecular mass: $228,984 \mathrm{Da}$ ) under nondenaturing conditions, as shown in Figure $5 \mathrm{~b}$. The native MS data also indicated that the higher-molecular-mass complexes 
observed for these mixtures under the present conditions corresponded to complexes composed of fourteen $\alpha 7$ subunits and one $\alpha 4$ or $\alpha 6$ subunit, indicating that $\alpha 4$ and $\alpha 6$ can bind to the $\alpha 7$ double ring.

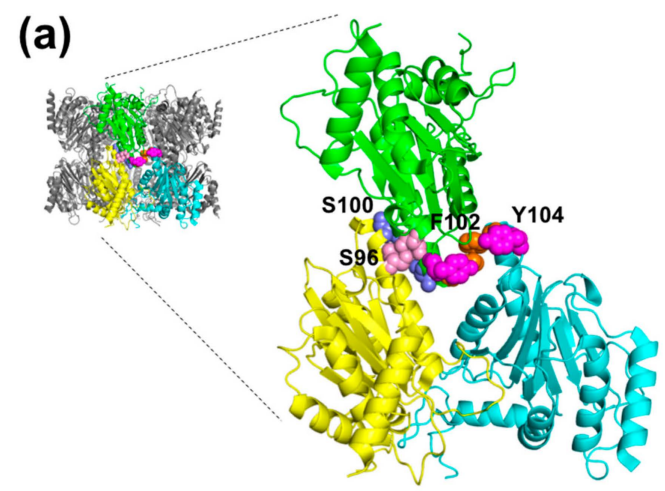

(b)

(c)
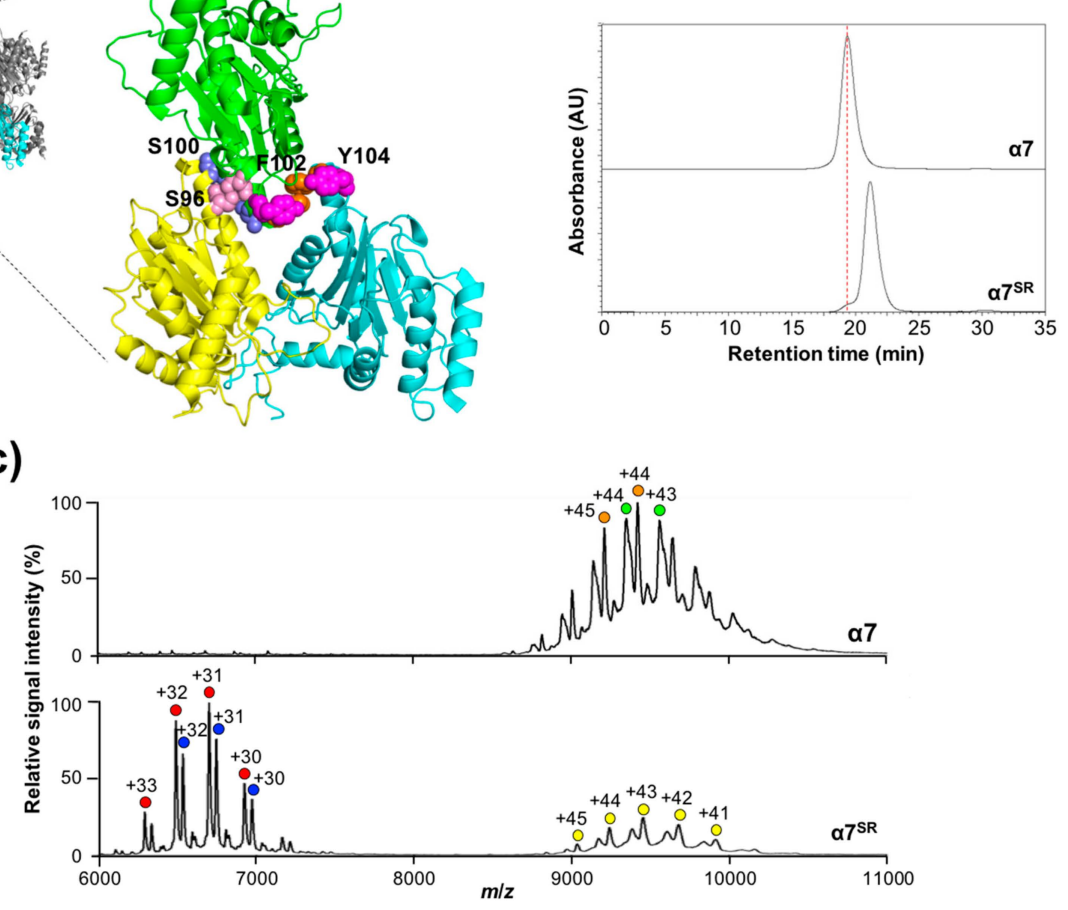

Figure 4. Generation of the single-ring $\alpha 7$ mutant. (a) Mutated positions of the single-ring mutant $\left(\alpha 7^{S R}\right)$. The mutated resides shown in sphere models are labeled in the close-up view. (b) Size-exclusion chromatogram of $\alpha 7^{\mathrm{SR}}$. Red dotted line indicates position of the SEC peak of $\alpha 7$ homotetradecamer. (c) Mass spectra of $\alpha 7^{\text {SR }}$ under nondenaturing conditions. Green and orange circles indicate the ion series of the $\alpha 7$ homotetradecamer. Blue and red circles indicate the ion series of the homoheptameric complex of $\alpha 7^{\mathrm{SR}}$. Yellow circles show those of the homotetradecameric complex of $\alpha 7^{\mathrm{SR}}$.

(a)

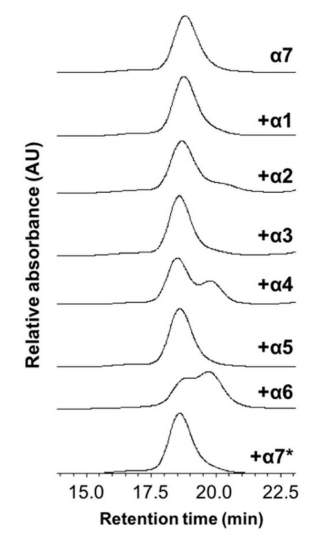

(b)

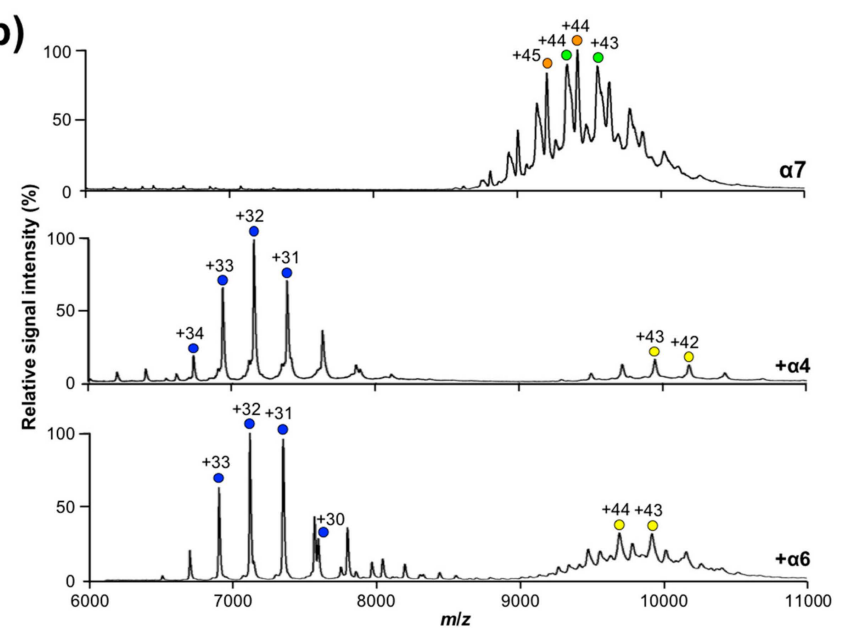

Figure 5. Evaluation of the $\alpha 7$-disassembling ability of the proteasome $\alpha$ subunits. (a) Size-exclusion chromatogram patterns of $\alpha 7$-containing fractions depending on the presence of the $\alpha 1-\alpha 6$ subunits and the $\alpha 7^{*}$ mutant (20 $\mu \mathrm{M}$ monomers). (b) Mass spectra of $\alpha 4 / \alpha 7$ and $\alpha 6 / \alpha 7$ mixtures under nondenaturing conditions. Green and orange circles indicate the ion series of the $\alpha 7$ homotetradecamer. Blue circles indicate the ion series of the 1:7 hetero-octameric complexes of $\alpha 4 / \alpha 7$ and $\alpha 6 / \alpha 7$, and yellow circles indicate those of the 1:14 heteropentadecameric complexes of $\alpha 4 / \alpha 7$ and $\alpha 6 / \alpha 7$. 
Our previous AFM data indicated that the disassembly of the $\alpha 7$ double ring by $\alpha 6$ involves two steps-the $\alpha 6$ monomer initially cracks at the interface between two stacked $\alpha 7$ single rings and subsequently occupies the central pore of the $\alpha 7$ single ring [26]. Therefore, we examined possible interactions of the $\alpha 7$ single ring with $\alpha 4$ as well as $\alpha 6$. Using the $\alpha 7^{S R}$ mutant, we performed SEC-based binding analysis with the $\alpha 1-\alpha 6$ subunits together with $\alpha 7^{*}$, as shown in Figure 6a. Sodium dodecyl sulfate polyacrylamide gel electrophoresis (SDS-PAGE) analysis detected the $\alpha 2, \alpha 4$, and $\alpha 6$ but not the $\alpha 1, \alpha 3, \alpha 5$, and $\alpha 7^{*}$ in the fractions co-eluted with $\alpha 7^{\mathrm{SR}}$ at approximately $21 \mathrm{~min}$. The mass spectra of the $\alpha 7^{\mathrm{SR}} / \alpha 2, \alpha 7^{\mathrm{SR}} / \alpha 4$, and $\alpha 7^{\mathrm{SR}} / \alpha 6$ complexes under nondenaturing conditions showed a major ion series indicating their 1:7 hetero-octameric complexes with molecular masses of 225,164 \pm 62 , $229,435 \pm 116$, and 228,717 $\pm 124 \mathrm{Da}$ (with theoretical masses of 225,230; 229,257; and 229,010 Da), respectively, as shown in Figure $6 \mathrm{~b}$. Regarding $\alpha 2$, MS peaks originating from the unbound $\alpha 7$ single ring were also observed, suggesting their weak interactions, as shown in Figure 6b. All these results indicate that $\alpha 4$ and $\alpha 6$, and to a lesser extent $\alpha 2$ but not the other $\alpha$ subunits, can interact with the disassembled $\alpha 7$ single ring in solution.

The cavitary surface of the homoheptameric ring of $\alpha 7$ exhibits unique charge distributions characterized by central negatively charged clusters surrounded by positively charged zones. The $\alpha 2$, $\alpha 4$, and $\alpha 6$ subunits commonly display positively and negatively charged clusters that are polarized near the vertex and at the base regions, respectively, of their triangle-shaped architecture; however, the negatively charged patch at the base region of $\alpha 2$ is smaller than those of $\alpha 4$ and $\alpha 6$, as shown in Figure S3. These charge complementarities may explain the selective accommodation of these $\alpha$ subunits in the cavity of the $\alpha 7$ homoheptameric ring. The double-ring cracking abilities of $\alpha 4$ and $\alpha 6$ may also be ascribed to their remarkably polarized charged clusters, although the structural basis of their transient interactions with the $\alpha 7$ double ring remains elusive.

It is plausible that the hetero-octameric complexes composed of the $\alpha 7$ and $\alpha 4$ (or $\alpha 6$ ) subunits cannot be re-associated into double rings because $\alpha 4$ as well as $\alpha 6$ occupies the central pore of the $\alpha 7$ homoheptameric ring, thereby sterically blocking inter-ring interactions. However, the hetero-octameric complexes are likely to be able to form complexes with unoccupied $\alpha 7$ homoheptameric rings, consequently encapsulating $\alpha 4$ and $\alpha 6$ within the $\alpha 7$ double-ring cage. One intriguing possibility is that these cages were detected as 14:1 $\alpha 7 / \alpha 4$ and 14:1 $\alpha 7 / \alpha 6$ complexes in the native mass spectra, as shown in Figure $5 b$.

\subsection{Creation of Heterotetradecameric Double-Ring Structures of the Proteasomal Subunits}

The self-assembling property of $\alpha 7$ suggests that proteasome formation involves some scrap-and-build mechanisms via which the $\alpha 7$ homotetradecamer is disassembled and the monomeric $\alpha 7$ subunit is integrated into the heteroheptameric $\alpha$ ring. To obtain insights into putative hetero-assembling processes involving $\alpha 7$, we used SEC to characterize possible interactions of the mutationally monomerized $\alpha 7^{*}$ with the other subunits, particularly $\alpha 1$ and $\alpha 6$, which flank $\alpha 7$ in the native heteroheptameric $\alpha$-ring. Of note, the results indicated that $\alpha 1, \alpha 4$, and $\alpha 6$, but not the other $\alpha$ subunits, are co-assembled with $\alpha 7^{*}$, giving rise to high-molecular-mass complexes of sizes comparable with that of the $\alpha 7$ homotetradecamer, as shown in Figure 7. The stoichiometry of the $\alpha 7^{*} / \alpha 1, \alpha 7^{*} / \alpha 4$, and $\alpha 7^{*} / \alpha 6$ hetero-oligomeric complexes was estimated as 1:3.6, 1.2:1, and 2.5:1, respectively, based on the Coomassie Brilliant Blue (CBB)-staining SDS-PAGE results, as shown in Figure 7.

AFM data of the high-molecular-mass complex fraction of $\alpha 7^{*}$ with $\alpha 4$ or $\alpha 6$ identified tetradecameric particles of double-ring shape along with particles of single heptameric rings and oligomeric structures without ring shape, which presumably resulted from the disruption of the double-ring oligomers on the mica surface, as shown in Figure 8a. As for the $\alpha 1 / \alpha 7^{*}$ complex, tetradecameric double rings and heptameric single rings were barely detected in the AFM observation possibly owing to their unstable structures on the mica surface. Instead, oligomeric structures were observed with a height of $\sim 10 \mathrm{~nm}$, which is comparable with those of the $\alpha 7, \alpha 4 / \alpha 7^{*}$, and $\alpha 6 / \alpha 7^{*}$ tetradecamers $(\sim 9 \mathrm{~nm})$. To obtain a higher-resolution structure, the $\alpha 4 / \alpha 7^{*}$ hetero-oligomeric complex 
was subjected to negative-staining electron microscopy (EM), which also indicated that the complex had a double-ring tetradecameric structure, as shown in Figure 8b.

(a)
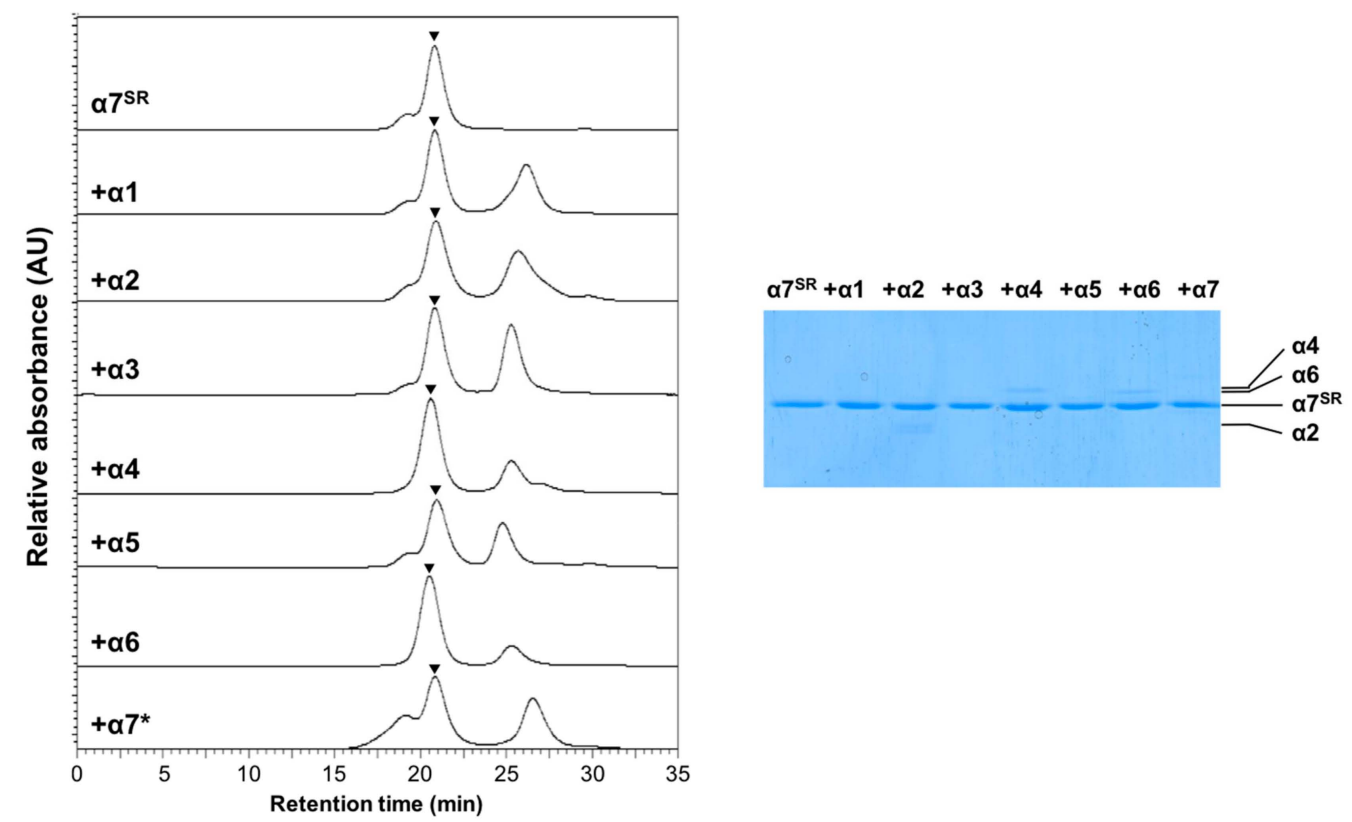

(b)

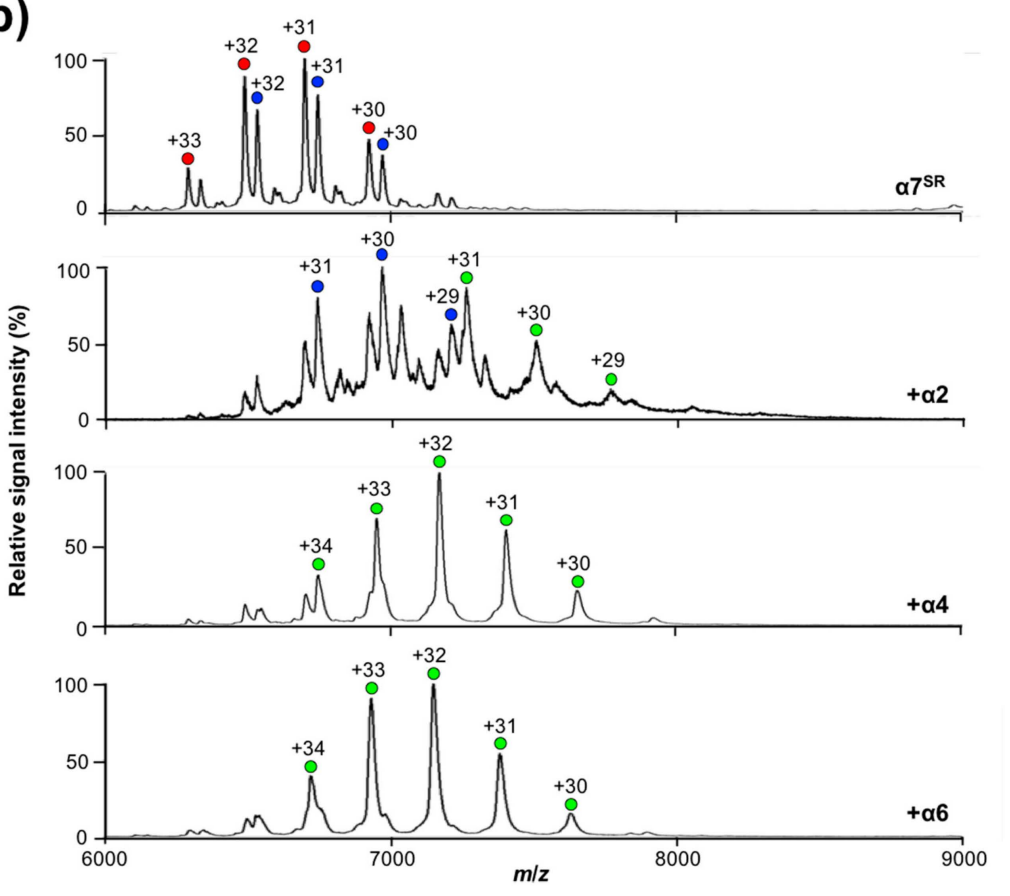

Figure 6. Examination of possible interactions of the $\alpha 7$ single ring and the proteasome $\alpha$ subunits. (a) Size-exclusion chromatogram of $\alpha 7^{\mathrm{SR}}$ in the presence of the $\alpha 1-\alpha 6$ subunits and the $\alpha 7^{*}$ mutant (20 $\mu \mathrm{M}$ monomers): SDS-PAGE of the size-exclusion chromatography (SEC) peaks (at approximately $21 \mathrm{~min}$ ) originating from the $\alpha 7^{\mathrm{SR}} / \alpha 1-\alpha 6$ and $\alpha 7^{*}$ complexes. The peak position is indicated by an inverted triangle. (b) Mass spectra of the $\alpha 7^{\mathrm{SR}} / \alpha 2, \alpha 7^{\mathrm{SR}} / \alpha 4$, and $\alpha 7^{\mathrm{SR}} / \alpha 6$ mixtures under nondenaturing conditions. Blue and red circles indicate major ion series of the $\alpha 7^{\mathrm{SR}}$. Green circles show the ion series of the 7:1 hetero-octameric complexes of $\alpha 7^{\mathrm{SR}} / \alpha 2, \alpha 7^{\mathrm{SR}} / \alpha 4$, and $\alpha 7^{\mathrm{SR}} / \alpha 6$ complexes. 


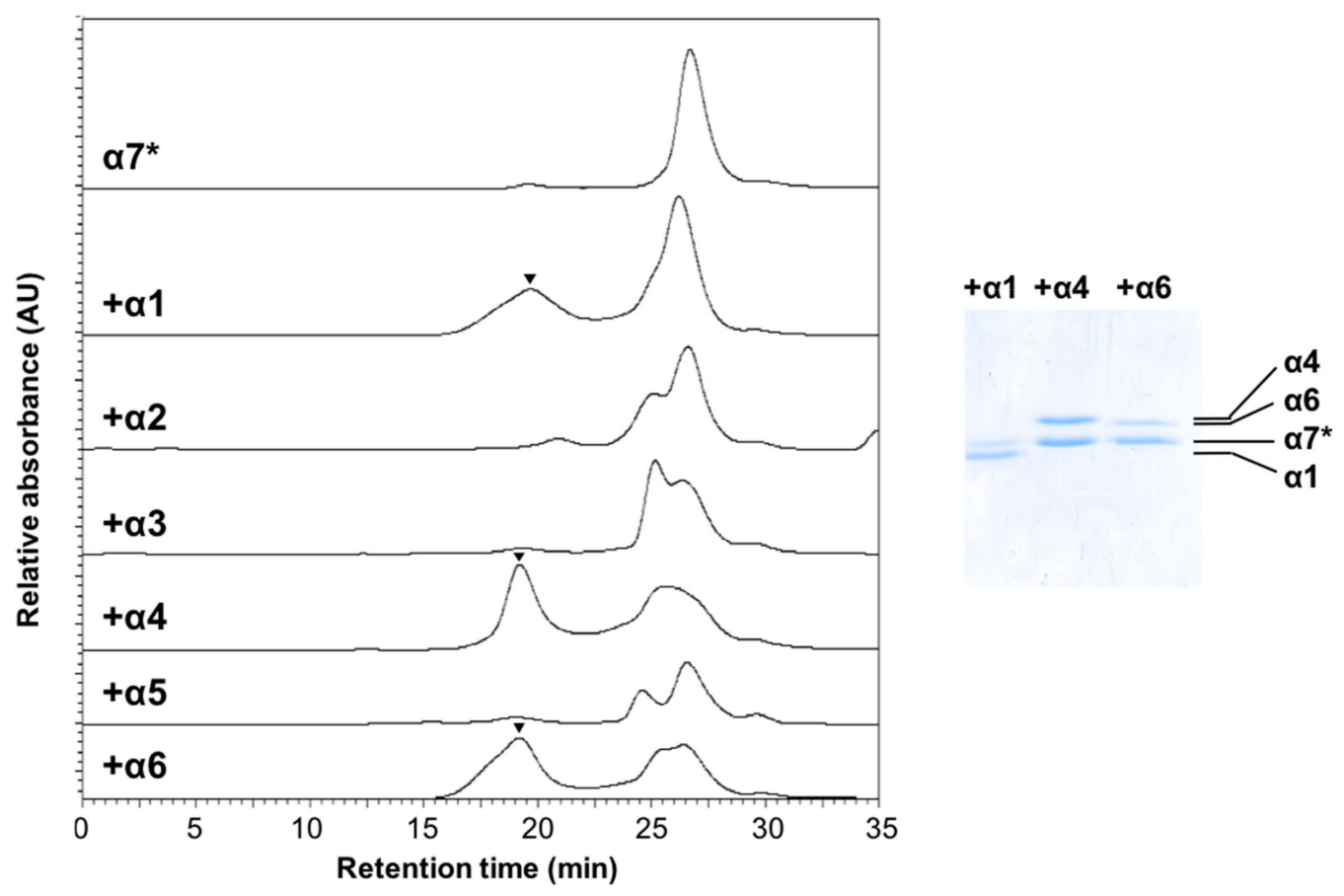

Figure 7. Exploration of possible formation of the $\alpha 7$ hetero-oligomeric complex mediated by proteasome $\alpha$ subunits. Size-exclusion chromatogram of $\alpha 7^{*}$ in the presence of $\alpha 1-\alpha 6$ subunits. The $\alpha 1-\alpha 6$ subunits ( $20 \mu \mathrm{M}$ monomers) were mixed with an equimolar amount of $\alpha 7^{*}$ at $20^{\circ} \mathrm{C}$ for $1 \mathrm{~h}$, and the mixtures were subsequently analyzed by SEC. The peak position is indicated by an inverted triangle. SDS-PAGE of the purified $\alpha 7^{*} / \alpha 1, \alpha 7^{*} / \alpha 4$, and $\alpha 7^{*} / \alpha 6$ complexes.

(a)

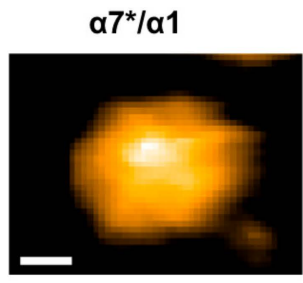

(b)

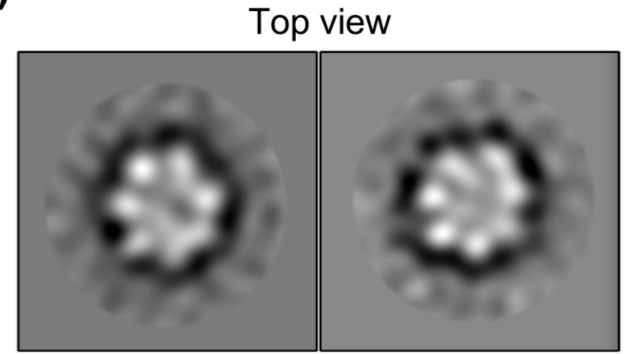

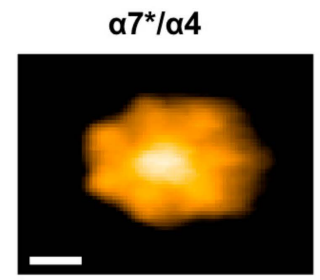

$\alpha 7^{\star} / \alpha 6$

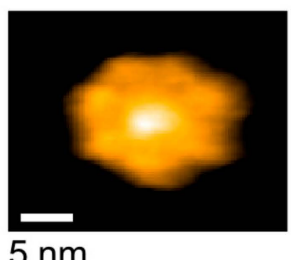

$5 \mathrm{~nm}$

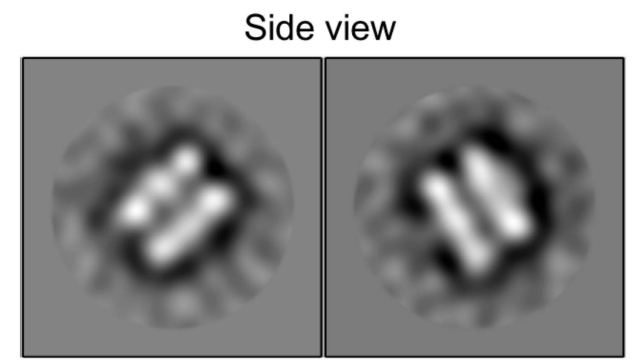

$10 \mathrm{~nm}$

Figure 8. Structural characterization of the $\alpha 7^{*} / \alpha 4$ and $\alpha 7^{*} / \alpha 6$ hetero-oligomeric complexes. (a) AFM images of two typical orientations of the $\alpha 7^{*} / \alpha 1$ (left), $\alpha 7^{*} / \alpha 4$ (center), and $\alpha 7^{*} / \alpha 6$ (right) complexes on bare mica. Scale bar: $5 \mathrm{~nm}$. (b) Two-dimensional averaged image of an $\alpha 7^{*} / \alpha 4$ particle subjected to single-particle negative-staining electron microscopy.

It is puzzling that mixture of the artificially monomerized subunit $\left(\alpha 7^{*}\right)$ and the intrinsically monomeric subunit ( $\alpha 4$ or $\alpha 6$ ) yields the heterotetradecameric double-ring architecture. One might 
assume that, for example, the $\alpha 6-\alpha 7^{*}$ interaction is stronger than the $\alpha 6-\alpha 6$ and $\alpha 7^{*}-\alpha 7^{*}$ interactions. However, such more favorable residue pair(s) could not be found by simply comparing the amino acid residues at the intersubunit interfaces, i.e., at regions $2-5$, based on the crystal structure of the $\alpha 7$ homotetradecamer, as shown in Figure S1 and Table S1. Rather, the crystallographic data highlight a small but significant difference between the $\alpha 7-\alpha 7$ contacting mode [25] and the $\alpha 6-\alpha 7$ interaction in the crystal structure of the human $20 S$ proteasome, as shown in Figure S4 [28]. Consequently, the overall quaternary structure of the $\alpha 7$ homoheptameric ring is markedly different from that of the native heteroheptameric $\alpha$ ring. This implies that accumulation of the slightly different contact mode at the subunit interface results in geometric frustration of the formation of the circular quaternary structure, thereby causing deformation or disruption of the ring structure. Based on the data obtained in this study, we suggest that not only local structural complementarity at the subunit interfaces but also geometric consistency and/or structural adjustability in terms of the formation of the circular structure are factors that determine the heptamerization of the proteasome $\alpha$ subunits. The present study demonstrates that geometric frustration can be compromised by combining structurally homologous protomers with potential but imperfect ability to self-assemble, thereby providing insights for controlling the assembly and disassembly of the proteasomal subunits. Furthermore, our findings would provide clues for the design and creation of circular hetero-oligomers based on the homo-oligomeric structural frameworks.

\section{Materials and Methods}

\subsection{Preparation of Wild-Type and Mutated Proteasome $\alpha$ Subunits}

Human proteasome $\alpha 6$ short isoform [PSMA1 (P25786); residues 1-263] and $\alpha 7-$ [PSMA3 (P25788); residues 1-255] subunits were expressed and purified as described previously $[23,24,26]$. Genes encoding proteasome $\alpha 1$ [PSMA6 (P60900); residues 1-246] and $\alpha 4$ [PSMA7 (P60900); residues 1-248] were subcloned into the NdeI and SalI sites of pET28b (Merck Millipore, Burlington, MA, USA), whereas the $\alpha 2$ gene [PSMA2 (P25787); residues 1-234] was subcloned into the NdeI and XhoI sites of pRSFDuet-1 vector (Merck Millipore). In addition, genes encoding $\alpha 3$ [PSMA4 (P25789); residues 1-261] and $\alpha 5$ [PSMA5 (P28066); residues 1-241] were subcloned into the BamHI and XhoI or SalI sites of modified pCold-I and pCold-GST vectors (TaKaRa Bio Inc., Kusatsu, Japan), respectively, which contain the TEV protease cleavage site preceding the target genes. Monomeric mutants of $\alpha 7$ (designated as $\alpha 7^{*}$ and $\alpha 7^{\mathrm{F} 14 \mathrm{~A}}$ ) were created via truncation of $22 \mathrm{~N}$-terminal residues or introduction of the F14A mutation, respectively. The mutated $\alpha 7$ genes were subcloned into the NdeI and XhoI sites of the pET28b vector. In contrast, the single-ring mutant $\alpha 7^{\text {SR }}$ was generated by introducing the S96D, S100D, F102R, and Y104R mutations using the wild-type construct in pRSFDuet-1. All expression plasmids were introduced into Escherichia coli BL21-CodonPlus (DE3)-RIL (Agilent Technologies, Santa Clara, CA, USA).

For producing recombinant proteins, the $E$. coli cells harboring the expression plasmids were grown in Luria-Bertani medium containing $15 \mu \mathrm{g} / \mathrm{mL}$ kanamycin or $50 \mu \mathrm{g} / \mathrm{mL}$ ampicillin. The $\alpha 7^{\text {SR }}$ mutant was purified as employed for the wild-type $\alpha 7$. Briefly, except for $\alpha 2$, the recombinant proteins were purified from the soluble fraction obtained by sonication and centrifugation. The resultant cell lysates were subjected to affinity chromatography $\left[\mathrm{Ni}^{+}\right.$-charged Chelating Sepharose or Glutathione Sepharose 4B (GE Healthcare, Chicago, IL, USA)], and further purified using anion-exchange (RESOURCE Q, GE Healthcare) and size-exclusion (HiLoad 26/60 Superdex 75 or 200 pg; GE Healthcare) columns. The $\alpha 2$ was purified from the inclusion bodies and refolded according to standard dilution methods using a buffer containing $20 \mathrm{mM}$ Tris- $\mathrm{HCl}$ (pH 8.0), $400 \mathrm{mM}$ L-arginine, $250 \mathrm{mM} \mathrm{NaSCN}, 1 \mathrm{mM}$ oxidized glutathione, and $5 \mathrm{mM}$ reduced glutathione. The refolded protein was further purified using a HiLoad 26/60 Superdex 75pg column (GE Healthcare). 


\subsection{Determination of Molecular Mass}

The molecular masses of the human wild-type proteasome $\alpha$ subunits $(\alpha 1-\alpha 7)$ and the mutated $\alpha 7$ proteins $\left(\alpha 7^{*}, \alpha 7^{\mathrm{F} 14 \mathrm{~A}}\right.$, and $\left.\alpha 7^{\mathrm{SR}}\right)$ were estimated using SEC and native MS. In SEC, the samples (0.3-10 $\mu \mathrm{M}$ ) were loaded onto a Superose 6 increase 10/300 GL column (GE Healthcare) with $20 \mathrm{mM}$ Tris-HCl ( $\mathrm{pH} 8.0$ ) and $150 \mathrm{mM} \mathrm{NaCl}$ at a flow rate of $0.75 \mathrm{~mL} / \mathrm{min}$. For calibrating the column, ribonuclease A (13.7 kDa), chymotrypsinogen A (25 kDa), ovalbumin (43 kDa), bovine serum albumin (67 kDa), conalbumin (75 kDa), aldolase (158 kDa), ferritin (440 kDa) (GE Healthcare), and blue dextran 2000 were used. The elution profiles were recorded as absorbance values at $280 \mathrm{~nm}$.

In native MS, buffer exchange of the purified $\alpha 1-\alpha 7$ proteins (30-50 $\mu \mathrm{M}$ monomers) was performed using $150 \mathrm{mM}$ ammonium acetate ( $\mathrm{pH}$ 6.8-8.0) with a Bio-Spin 6 column (Bio-Rad, Hercules, CA, USA). The buffer-exchanged samples (5-20 $\mu \mathrm{M}$ monomers) were immediately subjected to nanoflow electrospray ionization MS analysis with gold-coated glass capillaries made in house. Approximately 2-5 $\mu \mathrm{L}$ samples were loaded for each measurement. Buffer-exchanged $\alpha 4$ and $\alpha 6$ (4 $\mu \mathrm{M}$ monomers) were mixed with $\alpha 7$ ( $2 \mu \mathrm{M}$ tetradecamer) at $20^{\circ} \mathrm{C}$ for $1 \mathrm{~h}$ and subsequently analyzed using native MS. In contrast, $\alpha 4$ and $\alpha 6$ (4 $\mu \mathrm{M}$ monomers) were mixed with $\alpha 7^{\mathrm{SR}}$ mutant ( $4 \mu \mathrm{M}$ heptamer) and incubated as employed for wild-type $\alpha 7$. As for $\alpha 2$, the sample was incubated with $\alpha 7^{\mathrm{SR}}$ beforehand, and the buffer exchange was carried out for the mixture, and then subjected to native MS measurements. Spectra were acquired on a SYNAPT G2-Si HDMS mass spectrometer (Waters, Manchester, UK) in the positive ionization mode, as previously described [25]. Spectrum calibration was performed using 1 $\mathrm{mg} / \mathrm{mL}$ of cesium iodide and analysis was performed using the Mass Lynx software (Waters).

\section{3. $A F M$}

For AFM sample preparation, the $\alpha 1, \alpha 4$, or $\alpha 6$ (20 $\mu \mathrm{M}$ monomers) was mixed with an equal molar amount of $\alpha 7^{*}$ at $20^{\circ} \mathrm{C}$ for $1 \mathrm{~h}$, and subsequently fractionated by SEC. The high-molecular-mass complex fractions were subjected to the AFM analysis. AFM was performed using a laboratory-constructed apparatus with cantilevers ( $7 \mu \mathrm{m}$ long, $2 \mu \mathrm{m}$ wide, and $90 \mathrm{~nm}$ thick) at room temperature [29]. Typical values of the spring constant, resonant frequency, and quality factor of the cantilever in an aqueous solution are approximately $0.2 \mathrm{~N} / \mathrm{m}, 800 \mathrm{kHz}$, and 2, respectively. In AFM imaging, the free and set-point oscillation amplitudes were set to approximately $1.5 \mathrm{~nm}$ and $90 \%$ of the former, respectively. All samples were applied to either bare mica in $20 \mathrm{mM}$ Tris- $\mathrm{HCl}(\mathrm{pH} 8.0)$ with $150 \mathrm{mM} \mathrm{NaCl}$, as previously described [26].

\section{4. $E M$}

The protein samples for EM measurement were prepared using the same protocol as in the AFM analysis. Negative-staining EM was performed using a conventional protocol, as previously described [30]. EM imaging of the $\alpha 4 / \alpha 7^{*}$ hetero-oligomeric complex was performed at room temperature using a JEOL JEM 2200FS electron microscope (JEOL Ltd., Tokyo, Japan) equipped with a field emission gun operating at an acceleration voltage of $200 \mathrm{kV}$. A total of 40 images were obtained using a DE20 direct detection camera (Direct Electron LP, San Diego, CA, USA) at a detector magnification of 50,000 with an energy slit width of $20 \mathrm{eV}$ using the low-dose mode. The image size was set to $1.09 \AA$ per pixel on the camera. After subjection of motion collection with the DE_process_frames.py script, the obtained images were processed with Relion 2.0 software [31]. Subsequently, 1,534-particle images were extracted from the 40 images and subjected to two-dimensional classification after sorting with cross-correlation coefficients.

Supplementary Materials: Supplementary Materials can be found at http://www.mdpi.com/1422-0067/20/9/2308/ s1.

Author Contributions: T.S. (Taichiro Sekiguchi), T.S. (Tadashi Satoh), E.K., and K.K. conceived and designed the study; T.S. (Taichiro Sekiguchi), T.S. (Tadashi Satoh), E.K., H.Y., and S.Y. performed protein designing and sample preparation; T.S. (Taichiro Sekiguchi), K.I., and S.U. performed native MS; T.K., H.W., and T.U. performed AFM; C.S. and K.M. performed EM; T.S. (Tadashi Satoh) and K.K. mainly drafted the manuscript. 
Funding: This work was supported in part by the Grants-in-Aid for Scientific Research (Grant Numbers JP16H06280 to T.S., JP26460051 to E.K., JP17H05890 to H.Y., JP18H04512, JP18H01837 to T.U., and JP25102008, JP15H02491 to K.K.), by the Grants-in-Aid for Scientific Research on Innovative Areas-Platforms for Advanced Technologies and Research Resources, "Advanced Bioimaging Support" (JP16H06280), from the Ministry of Education, Culture, Sports, Science and Technology, Japan. This work was also supported by the Joint Studies Program in the Okazaki BIO-NEXT project of the Okazaki Institute for Integrative Bioscience (No. 303), by the Joint Research by Exploratory Research Center on Life and Living Systems (ExCELLS) (ExCELLS program No. 18-101 to T.U., and No. 18-402 to H.Y.), by Functional Genomics Facility, NIBB Core Research Facilities, and by SOKENDAI (The Graduate University for Advanced Studies).

Acknowledgments: The authors would like to thank Kumiko Hattori and Kiyomi Senda for their help in the preparation of recombinant proteins, Hiroki Kawamura for his contribution during the early stage of this study, and Michiko Nakano (Institute for Molecular Science) for constructing three-dimensional protein models useful for insightful discussion.

Conflicts of Interest: The authors declare no conflict of interest.

\section{Abbreviations}

$\begin{array}{ll}\text { AFM } & \text { atomic force microscopy } \\ \text { CBB } & \text { Coomassie Brilliant Blue } \\ \text { EM } & \text { electron microscopy } \\ \text { MS } & \text { mass spectrometry } \\ m / z & \text { mass-to-charge ratio } \\ \text { PDB } & \text { Protein Data Bank } \\ \text { SDS-PAGE } & \text { sodium dodecyl sulfate polyacrylamide gel electrophoresis } \\ \text { SEC } & \text { size-exclusion chromatography } \\ \text { SR } & \text { single-ring }\end{array}$

\section{References}

1. Garcia-Bellido, A. Symmetries throughout organic evolution. Proc. Natl. Acad. Sci. USA 1996, 93, 14229-14232. [CrossRef]

2. Goodsell, D.S.; Olson, A.J. Structural symmetry and protein function. Annu. Rev. Biophys. Biomol. Struct. 2000, 29, 105-153. [PubMed]

3. Snider, J.; Houry, W.A. AAA+ proteins: Diversity in function, similarity in structure. Biochem. Soc. Trans. 2008, 36, 72-77.

4. Lin, Y.R.; Koga, N.; Vorobiev, S.M.; Baker, D. Cyclic oligomer design with de novo $\alpha \beta$-proteins. Protein Sci. 2017, 26, 2187-2194. [CrossRef] [PubMed]

5. Strauch, E.M.; Bernard, S.M.; La, D.; Bohn, A.J.; Lee, P.S.; Anderson, C.E.; Nieusma, T.; Holstein, C.A.; Garcia, N.K.; Hooper, K.A.; et al. Computational design of trimeric influenza-neutralizing proteins targeting the hemagglutinin receptor binding site. Nat. Biotechnol. 2017, 35, 667-671. [CrossRef]

6. Tanaka, K. The proteasome: Overview of structure and functions. Proc. Jpn. Acad. Ser. B Phys. Biol. Sci. 2009, 85, 12-36. [CrossRef] [PubMed]

7. Collins, G.A.; Goldberg, A.L. The Logic of the 26S Proteasome. Cell 2017, 169, 792-806. [CrossRef]

8. Kato, K.; Satoh, T. Structural insights on the dynamics of proteasome formation. Biophys. Rev. 2018, 10, 597-604. [CrossRef] [PubMed]

9. Löwe, J.; Stock, D.; Jap, B.; Zwickl, P.; Baumeister, W.; Huber, R. Crystal structure of the 20S proteasome from the archaeon T. acidophilum at $3.4 \AA$ resolution. Science 1995, 268, 533-539.

10. Unno, M.; Mizushima, T.; Morimoto, Y.; Tomisugi, Y.; Tanaka, K.; Yasuoka, N.; Tsukihara, T. The structure of the mammalian $20 \mathrm{~S}$ proteasome at $2.75 \AA$ resolution. Structure 2002, 10, 609-618. [CrossRef]

11. Kish-Trier, E.; Hill, C.P. Structural biology of the proteasome. Annu. Rev. Biophys. 2013, 42, 29-49. [CrossRef] [PubMed]

12. Finley, D.; Chen, X.; Walters, K.J. Gates, Channels, and Switches: Elements of the Proteasome Machine. Trends Biochem. Sci. 2016, 41, 77-93. [CrossRef] [PubMed]

13. Budenholzer, L.; Cheng, C.L.; Li, Y.; Hochstrasser, M. Proteasome Structure and Assembly. J. Mol. Biol. 2017, 429, 3500-3524. [CrossRef] [PubMed] 
14. Sprangers, R.; Kay, L.E. Quantitative dynamics and binding studies of the $20 \mathrm{~S}$ proteasome by NMR. Nature 2007, 445, 618-622. [CrossRef] [PubMed]

15. Yashiroda, H.; Mizushima, T.; Okamoto, K.; Kameyama, T.; Hayashi, H.; Kishimoto, T.; Niwa, S.; Kasahara, M.; Kurimoto, E.; Sakata, E.; et al. Crystal structure of a chaperone complex that contributes to the assembly of yeast 20 S proteasomes. Nat. Struct. Mol. Biol. 2008, 15, 228-236. [CrossRef]

16. Murata, S.; Yashiroda, H.; Tanaka, K. Molecular mechanisms of proteasome assembly. Nat. Rev. Mol. Cell Biol. 2009, 10, 104-115. [CrossRef] [PubMed]

17. Takagi, K.; Saeki, Y.; Yashiroda, H.; Yagi, H.; Kaiho, A.; Murata, S.; Yamane, T.; Tanaka, K.; Mizushima, T.; Kato, K. $\mathrm{Pba} 3-\mathrm{Pba} 4$ heterodimer acts as a molecular matchmaker in proteasome $\alpha$-ring formation. Biochem. Biophys. Res. Commun. 2014, 450, 1110-1114. [CrossRef] [PubMed]

18. Kock, M.; Nunes, M.M.; Hemann, M.; Kube, S.; Dohmen, R.J.; Herzog, F.; Ramos, P.C.; Wendler, P. Proteasome assembly from $15 \mathrm{~S}$ precursors involves major conformational changes and recycling of the Pba1-Pba2 chaperone. Nat. Commun. 2015, 6, 6123. [CrossRef]

19. Kurimoto, E.; Satoh, T.; Ito, Y.; Ishihara, E.; Okamoto, K.; Yagi-Utsumi, M.; Tanaka, K.; Kato, K. Crystal structure of human proteasome assembly chaperone PAC4 involved in proteasome formation. Protein Sci. 2017, 26, 1080-1085. [CrossRef]

20. Wu, W.; Sahara, K.; Hirayama, S.; Zhao, X.; Watanabe, A.; Hamazaki, J.; Yashiroda, H.; Murata, S. PAC1-PAC2 proteasome assembly chaperone retains the core $\alpha 4-\alpha 7$ assembly intermediates in the cytoplasm. Genes Cells 2018, 23, 839-848. [CrossRef]

21. Satoh, T.; Yagi-Utsumi, M.; Okamoto, K.; Kurimoto, E.; Tanaka, K.; Kato, K. Molecular and Structural Basis of the Proteasome $\alpha$ Subunit Assembly Mechanism Mediated by the Proteasome-Assembling Chaperone PAC3-PAC4 Heterodimer. Int. J. Mol. Sci. 2019, 20, 2231. [CrossRef]

22. Gerards, W.L.; Enzlin, J.; Häner, M.; Hendriks, I.L.; Aebi, U.; Bloemendal, H.; Boelens, W. The human $\alpha$-type proteasomal subunit $\mathrm{HsC} 8$ forms a double ringlike structure, but does not assemble into proteasome-like particles with the $\beta$-type subunits HsDelta or HsBPROS26. J. Biol. Chem. 1997, 272, 10080-10086. [CrossRef] [PubMed]

23. Sugiyama, M.; Hamada, K.; Kato, K.; Kurimoto, E.; Okamoto, K.; Morimoto, Y.; Ikeda, S.; Naito, S.; Furusaka, M.; Itoh, K.; et al. SANS simulation of aggregated protein in aqueous solution. Nucl. Instrum. Methods Phys. Res. A 2009, 600, 272-274. [CrossRef]

24. Sugiyama, M.; Kurimoto, E.; Yagi, H.; Mori, K.; Fukunaga, T.; Hirai, M.; Zaccai, G.; Kato, K. Kinetic asymmetry of subunit exchange of homooligomeric protein as revealed by deuteration-assisted small-angle neutron scattering. Biophys. J. 2011, 101, 2037-2042. [CrossRef] [PubMed]

25. Ishii, K.; Noda, M.; Yagi, H.; Thammaporn, R.; Seetaha, S.; Satoh, T.; Kato, K.; Uchiyama, S. Disassembly of the self-assembled, double-ring structure of proteasome $\alpha 7$ homo-tetradecamer by $\alpha 6$. Sci. Rep. 2015, 5, 18167. [CrossRef] [PubMed]

26. Kozai, T.; Sekiguchi, T.; Satoh, T.; Yagi, H.; Kato, K.; Uchihashi, T. Two-step process for disassembly mechanism of proteasome $\alpha 7$ homo-tetradecamer by $\alpha 6$ revealed by high-speed atomic force microscopy. Sci. Rep. 2017, 7, 15373. [CrossRef]

27. Groll, M.; Brandstetter, H.; Bartunik, H.; Bourenkow, G.; Huber, R. Investigations on the maturation and regulation of archaebacterial proteasomes. J. Mol. Biol. 2003, 327, 75-83. [CrossRef]

28. Schrader, J.; Henneberg, F.; Mata, R.A.; Tittmann, K.; Schneider, T.R.; Stark, H.; Bourenkov, G.; Chari, A. The inhibition mechanism of human $20 \mathrm{~S}$ proteasomes enables next-generation inhibitor design. Science 2016, 353, 594-598. [CrossRef]

29. Uchihashi, T.; Kodera, N.; Ando, T. Guide to video recording of structure dynamics and dynamic processes of proteins by high-speed atomic force microscopy. Nat. Protoc. 2012, 7, 1193-1206. [CrossRef]

30. Murata, K.; Nishimura, S.; Kuniyasu, A.; Nakayama, H. Three-dimensional structure of the $\alpha 1-\beta$ complex in the skeletal muscle dihydropyridine receptor by single-particle electron microscopy. J. Electron. Microsc. (Tokyo) 2010, 59, 215-226. [CrossRef]

31. Scheres, S.H. RELION: Implementation of a Bayesian approach to cryo-EM structure determination. J. Struct. Biol. 2012, 180, 519-530. [CrossRef] [PubMed]

(C) 2019 by the authors. Licensee MDPI, Basel, Switzerland. This article is an open access article distributed under the terms and conditions of the Creative Commons Attribution (CC BY) license (http://creativecommons.org/licenses/by/4.0/). 